\title{
A thorough study of Si nanowire FETs with 3D Multi-Subband Ensemble Monte Carlo simulations
}

\author{
L. Donetti*, C. Sampedro, F.G. Ruiz, A. Godoy, F. Gamiz \\ Departamento de Electrónica and CITIC, Universidad de Granada, 18071 Granada, Spain.
}

\begin{abstract}
In this paper, the DC electrical behavior of n-MOS transistors based on Si nanowires with $\langle 100\rangle$ and $\langle 110\rangle$ channel orientations is thoroughly compared by means of MultiSubband Ensemble Monte Carlo simulations. We find that the drain current depends on the nanowire diameter and it is slightly, but consistently, larger for $\langle 100\rangle$ than for $\langle 110\rangle$ nanowires. The observed differences in mobility, velocity and spatial charge distribution are interpreted in terms of the effective masses and populations of the different Si conduction band valleys, whose six-fold degeneracy is lifted by quantum confinement in narrow nanowires. Finally, we study the scaling behavior for channel lengths down to $8 \mathrm{~nm}$, concluding that the differences observed between both orientations are minimal.
\end{abstract}

Keywords: Gate-all-around MOSFET, Monte Carlo simulation, multi-subband, short-channel effects, nanowire orientation.

\section{Introduction}

Scaling the channel length of Field-Effect Transistors (FETs) in the nanometer range is not trivial if we strive to keep short channel effects under control. To achieve this goal, a strong electrostatic control of the gate electrode over the channel charge is required. The best possible electrostatic control is obtained when the channel is completely surrounded by the gate, as in the Gate-All-Around (GAA) devices [1]. In this

\footnotetext{
*Corresponding author

Email address: donetti@ugr.es (L. Donetti)
} 
paper, we consider GAA transistors based on cylindrical Si nanowires with different crystal orientations and sub-10 $\mathrm{nm}$ diameter. When different nanowire orientations are considered, it is found that $\langle 100\rangle$ oriented devices show larger electron mobility [2] and higher ballistic conductance [3]. However, the performance of very short channel devices cannot be predicted only based on these parameters as a comprehensive device description is needed, especially to assess the scaling perspective and the short channel effects. The comparison between $\langle 100\rangle$ and $\langle 110\rangle$ nanowires is especially relevant: a potentially better performance of the former configuration with respect to the latter would pose a fabrication challenge to integrate nanowire-based transistors with traditional bulk FETs or FinFETs with $\langle 110\rangle$ channel orientation.

To perform such a study, we employ an in-house developed 3D Multi-Subband Ensemble Monte Carlo simulator [4], which takes into account the quantum effects of $2 \mathrm{D}$ confinement, thanks to the solution of the $2 \mathrm{D}$ Schrödinger equation in several cross sections of the device, and employs Monte Carlo (MC) method, able to model non-equilibrium transport and compute the $I-V$ characteristics. The simulator has already proved its capabilities for the analysis of $3 \mathrm{D}$ devices where $2 \mathrm{D}$ confinement is important, such as Si nanowires [4] and FinFETs with small cross section [5].

The outline of this paper is as follows: in section 2 we briefly describe the simulator, in section 3 we define the structure of the devices under study and discuss the results and, finally, in section 4 we draw the main conclusions.

\section{Simulation setup}

The 3D simulation tool employed in this work is based on the space-mode approach and has been described in detail in previous publications $[4,5]$. Its main feature with respect to classical or quantum-corrected MC simulators is that quantum confinement in the plane perpendicular to electron transport is fully taken into account, by solving the 2D Schrödinger equation in several cross sections along the device. For transport, the simulator solves the 1D Boltzmann equation through the Ensemble MC method, taking the solutions of the Schrödinger equation as inputs: subband energy profiles determine the drift field and wave-functions are employed to compute the scattering 
rates. Finally, self consistency is achieved by coupling this procedure in a loop with the solution of the 3D Poisson equation.

The structure of the device under study is described employing a 3D finite element mesh with tetrahedral elements. As we need to solve the Schrödinger equation in different cross sections of the device, this $3 \mathrm{D}$ mesh is constructed by extruding a $2 \mathrm{D}$ triangular mesh. The choice of finite elements allows a good representation of complex geometries and is particularly appropriate for the modeling of rounded devices such as the cylindrical nanowires studied in the present work. Electronic band structure is described employing the effective mass model with anisotropic valleys and nonparabolic corrections [6]. For very narrow nanowires, this description is not as accurate as the one that could be obtained employing multiple-band $\boldsymbol{k} \cdot \boldsymbol{p}$ models or atomistic ones such as those based on the Density Functional Theory or Tight Binding approach. However, the effective mass model can provide reliable results $[7,8]$ employing bulk values of the effective masses for Si nanowires down to a diameter of $5 \mathrm{~nm}$ and even below, if these values are correctly fitted [8]. The 1D MC simulation includes carrier scattering by acoustic and optical phonons [9], taking into account Pauli exclusion principle [10]. A variance reduction technique based on non-uniform super-particle weight [11] is employed in order to improve the MC statistics, reducing the statistical noise in the sub-threshold regime: the weight of a simulation super-particle is computed according to its total energy [4]. To improve the performance of the simulator, a high level of parallelism is employed in the MC simulation and in the solution of Poisson and Schrödinger equations.

The same simulator also allows the calculation of carrier mobility. In this case only the channel of the device is considered, where a uniform electric field, $F_{z}$, is applied in the longitudinal direction, $z$, and the average electron velocity, $v_{z}$, is computed. Electron mobility is then extracted by fitting the curve of $v_{z}$ vs. $F_{z}$ in the limit of small field. 


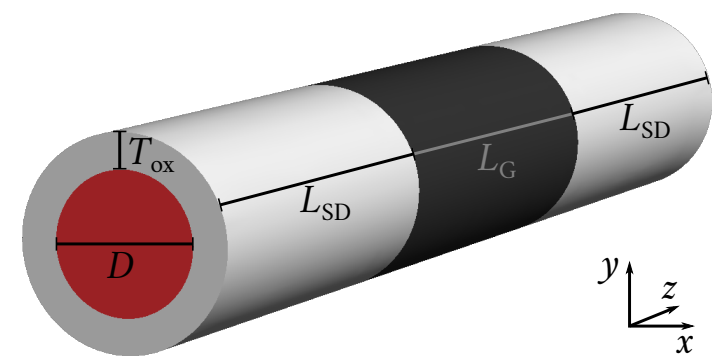

Figure 1: Structure of the devices simulated in this work. Device cross sections are parallel to the $x y$ plane, transport is along $z$ axis. The total length of the simulation domain in the $z$ direction is $L_{z}=L_{\mathrm{G}}+2 L_{\mathrm{SD}}$.

\section{Results and discussion}

The devices considered in this paper are GAA n-MOSFETs based on cylindrical Si nanowires with channel along either the $\langle 100\rangle$ or $\langle 110\rangle$ directions. Three different nanowire diameters are employed in this work: $D=4 \mathrm{~nm}, 6 \mathrm{~nm}$ and $8 \mathrm{~nm}$. When not explicitly stated otherwise, the channel length is $L_{\mathrm{G}}=14 \mathrm{~nm}$ and in the final scaling study $L_{\mathrm{G}}$ is reduced down to $8 \mathrm{~nm}$. The thickness of the $\mathrm{SiO}_{2}$ gate oxide is $T_{\mathrm{ox}}=1 \mathrm{~nm}$, the channel is considered undoped and a midgap metal is employed as gate material. The doping density in source and drain regions is set to $N_{\mathrm{SD}}=1 \times 10^{20} \mathrm{~cm}^{-3}$, with an underlap of $\Delta_{\mathrm{SD}}=2 \mathrm{~nm}$ from the gate edges and a Gaussian distribution with $\sigma_{\mathrm{SD}}=$ $0.8 \mathrm{~nm}$. The simulation domain includes source and drain regions for a length of $L_{\mathrm{SD}}=$ $14 \mathrm{~nm}$ each. The complete structure is depicted in Figure 1.

We first compute the transfer characteristics for the devices with the three values of the diameter and the two channel orientations, shown in Figure 2 for both low $\left(V_{\mathrm{D}}=\right.$ $50 \mathrm{mV})$ and high $\left(V_{\mathrm{D}}=0.5 \mathrm{~V}\right)$ values of drain bias. Before analyzing the results, we can observe that the statistical noise intrinsic to the MC procedure is evident only for values of $I_{\mathrm{D}}$ lower than $1 \mathrm{nA}$, thanks to the implementation of a variance reduction technique as previously mentioned. The trends we can observe in Figure 2 are the same for both values of $V_{\mathrm{D}}$. First, $I_{\mathrm{D}}$ decreases as the nanowires diameter is reduced. This is the result of two cumulative effects: $i$ ) narrower devices have higher threshold voltage, $V_{\mathrm{th}}$, because of stronger confinement which increases the subband energy levels, including the fundamental one; ii) narrower devices have smaller gate oxide capacitance per unit 


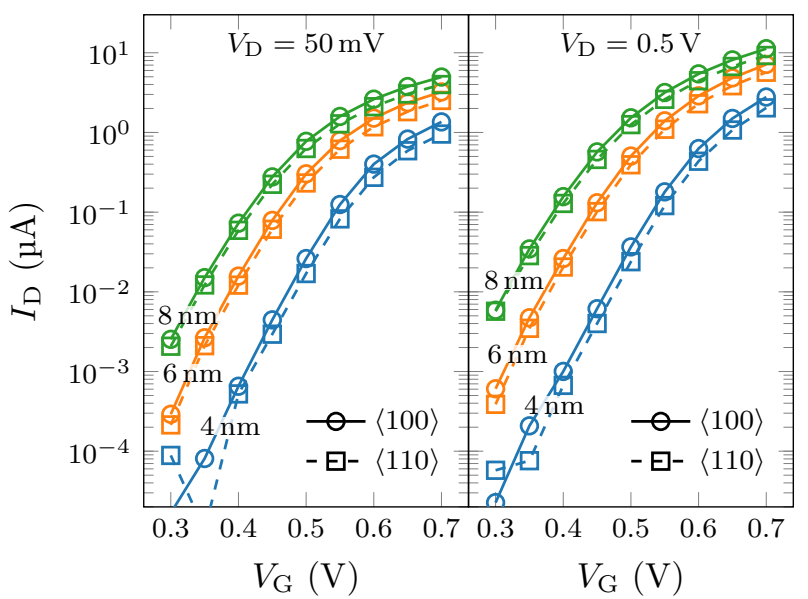

Figure 2: Transfer characteristics of $\langle 100\rangle$ and $\langle 110\rangle$ devices with different diameters, for $V_{\mathrm{D}}=50 \mathrm{mV}$ (left) and $V_{\mathrm{D}}=0.5 \mathrm{~V}$ (right).

length and therefore lower carrier density for the same gate overdrive voltage. Second, comparing the curves corresponding to different channel orientations, we observe that $\langle 100\rangle$ devices show a systematic larger current: the difference is not substantial, but it is noticeable in the whole range of gate bias and consistent for different values of the drain bias and nanowire diameter.

To check whether the difference between $\langle 100\rangle$ and $\langle 110\rangle$ devices is due to electrostatics or to transport properties, we first consider the inversion charge under the gate, which is shown in Figure 3, as a function of $V_{\mathrm{G}}$ for $V_{\mathrm{D}}=50 \mathrm{mV}$ for all devices. Here we can notice the same dependence on $D$ as observed before for the current, but now the difference between both channel orientations has essentially disappeared. As another check, we compare the $I_{\mathrm{D}}-V_{\mathrm{G}}$ curves by factoring out the electrostatic effects. To do that, we rescale the drain current to take into account the device geometry. For planar devices, this would simply mean dividing $I_{\mathrm{D}}$ by the channel width $W$. Instead, for $3 \mathrm{D}$ devices, the correct normalization involves the gate capacitance per unit length that, for cylindrical nanowires, is given by [12]:

$$
\frac{C_{\mathrm{ox}}(D)}{L}=\frac{2 \pi \kappa \epsilon_{0}}{\log \left(1+2 T_{\mathrm{ox}} / D\right)}
$$

where $\kappa$ is the dielectric constant of the oxide material and $\epsilon_{0}$ is the permittivity of 


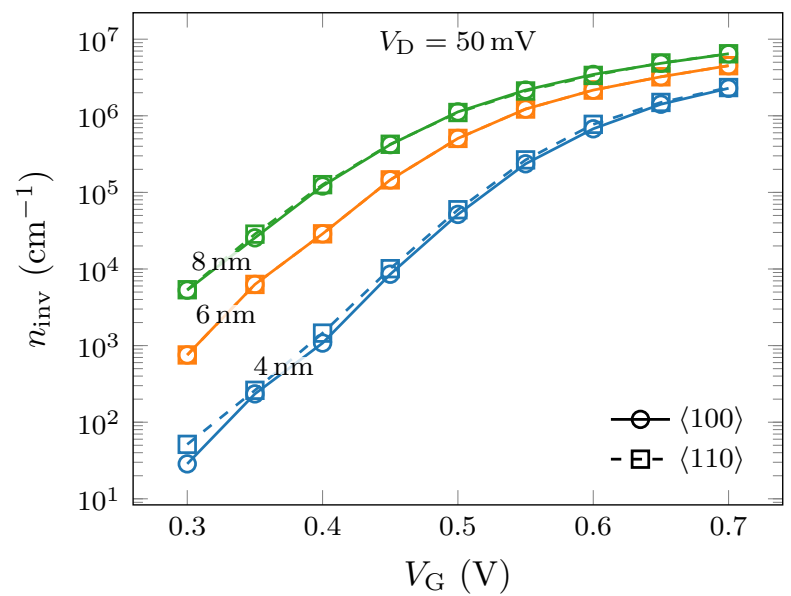

Figure 3: Linear inversion charge in the center of the devices with $\langle 100\rangle$ and $\langle 110\rangle$ and different diameters, for $V_{\mathrm{D}}=50 \mathrm{mV}$.

free space. Thus, we rescale the drain currents of the devices with different $D$ as if they all corresponded to a nanowire with $D=4 \mathrm{~nm}$, obtaining the scaled current $I_{\mathrm{D}, s}=I_{\mathrm{D}} \cdot\left(C_{\mathrm{ox}}(4 \mathrm{~nm}) / C_{\mathrm{ox}}(D)\right)$. Then, $I_{\mathrm{D}, s}$ is plotted against the gate overdrive voltage, that is $V_{\mathrm{G}}-V_{\text {th }}$. The threshold voltage, $V_{\text {th }}$, is extracted employing a fixed value of the normalized current, $I_{\mathrm{D}, s}=0.02 \mu \mathrm{A}$. Figure 4 shows the resulting scaled transfer characteristics for $V_{\mathrm{D}}=50 \mathrm{mV}$ : all the curves are essentially superposed in the subthreshold regime indicating that electrostatic effects have been correctly factored out. On the other hand, a larger current can still be observed for $\langle 100\rangle$ devices for large overdrive voltage. Therefore, we cannot ascribe such difference to electrostatics and we turn to the analysis of transport quantities, such as velocity and mobility.

The average electron velocity in the transport direction, $v_{z}$, is shown in Figure 5 as a function of position, $z$, along the channel, for a fixed overdrive voltage $V_{\mathrm{G}}-V_{\text {th }}=0.3 \mathrm{~V}$, with both small $\left(V_{\mathrm{D}}=50 \mathrm{mV}\right)$ and large $\left(V_{\mathrm{D}}=0.5 \mathrm{~V}\right)$ drain bias. It should be noticed that, for all considered nanowire diameters and drain biases, velocity is larger in $\langle 100\rangle$ than in $\langle 110\rangle$ channels. Now, we look separately at the results obtained for the two values of $V_{\mathrm{D}}$. For low drain bias the longitudinal field is relatively small: transport is near equilibrium. In this case, we can observe that the velocity inside the channel gets higher for wider nanowires and the position of the peak velocity moves from the drain 


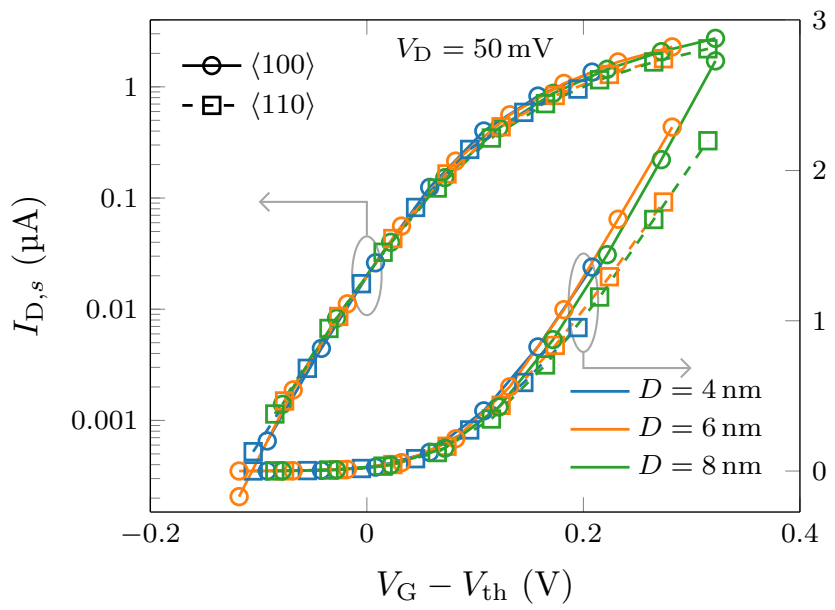

Figure 4: Normalized drain current $I_{\mathrm{D}, s}$ versus overdrive voltage for $\langle 100\rangle$ and $\langle 110\rangle$ devices with different diameters. All curves are computed for $V_{\mathrm{D}}=50 \mathrm{mV}$.

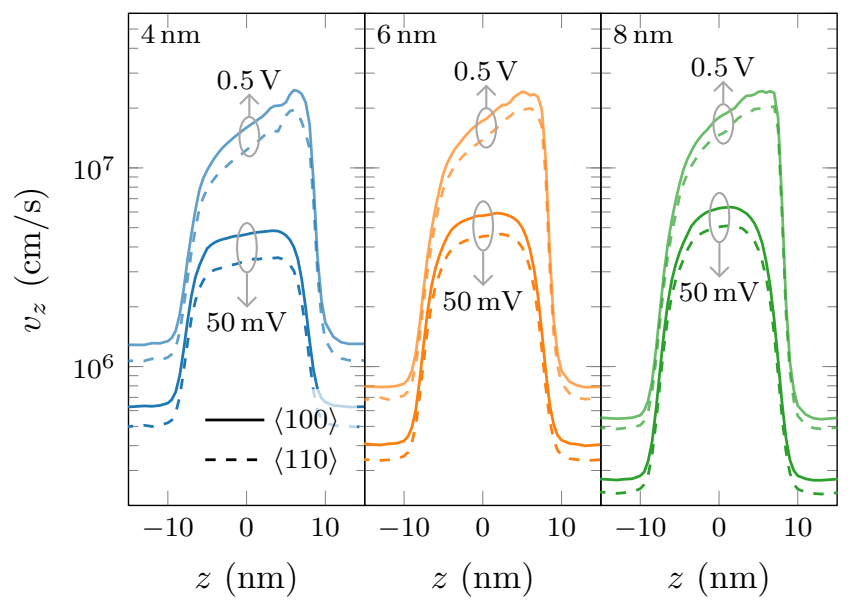

Figure 5: Ensemble average of the longitudinal electron velocity as a function of position $z$ along the channel for the different diameters. The voltages indicated by arrows are the corresponding values of $V_{\mathrm{D}}$. 


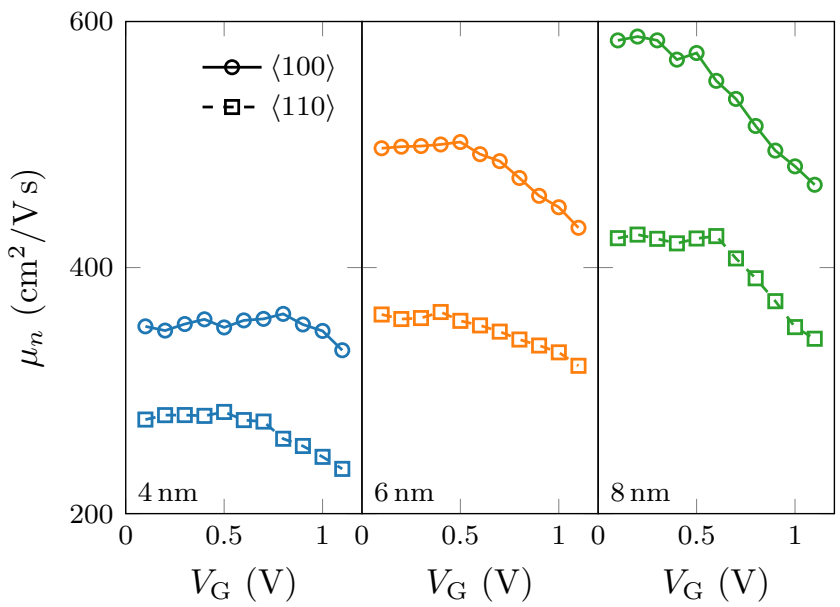

Figure 6: Electron mobility as a function of gate bias for the different orientations and nanowire diameters.

end of the channel towards its center. On the contrary, for high drain bias, transport is strongly out of equilibrium: now the shape and height of the velocity peaks in the channel is very similar for all considered values of $D$.

Next, we turn out attention to the electron mobility $\mu_{n}$, computed for the same channel structures but now assuming infinite channel length in the limit of small longitudinal electric field. In Figure 6 we can observe that $\mu_{n}$ always decreases as the gate bias increases due to stronger carrier confinement: phonon scattering is larger when the electron wave-functions are localized in smaller regions [13]. However, such dependence is weaker in the case of the narrowest nanowire with $D=4 \mathrm{~nm}$, because confinement in this case is essentially generated by the silicon/oxide barriers and only weakly modified by the gate field. The increased geometrical confinement is the reason for the reduction of $\mu_{n}$ for narrower nanowires. In any case, consistently with [2], mobility in $\langle 100\rangle$ channels is significantly larger than in $\langle 110\rangle$ channels for all considered nanowire sizes, with up to $40 \%$ difference. These observations are consistent with the conclusions we drew in the analysis of velocity for low $V_{\mathrm{D}}$ : in both cases transport is near equilibrium. Summing up, we can conclude that transport properties of $\langle 100\rangle$ nanowires are better than those of $\langle 110\rangle$ nanowires, both in near equilibrium and with a strong longitudinal field. 


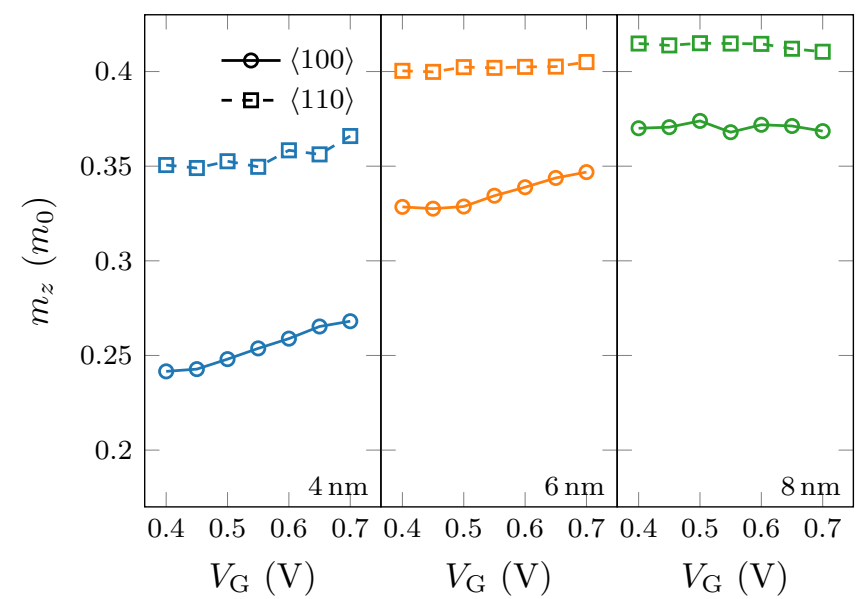

Figure 7: Average of electron effective transport mass in the middle of the channel as a function of gate bias for the different orientations and nanowire diameters.

This conclusion can be explained by analyzing the average of the effective transport mass, $m_{z}$, in the middle of the channel. This is computed as the average of the effective transport mass of each valley, weighted by the corresponding population. shown in Figure 7. Indeed, $m_{z}$ is smaller in nanowires with $\langle 100\rangle$ orientation that in those with $\langle 110\rangle$ channel. The larger mass of wider nanowires does not translate into a lower velocity or mobility because larger $D$ also imply a decrease of the scattering rates, as we already mentioned. The difference in effective mass for the two orientations stems from the different splitting of the six conduction bands of silicon (equivalent in the bulk semiconductor) due to quantum confinement. In $\langle 100\rangle$ nanowires, there is a set of four valleys with the longitudinal mass, $m_{l}=0.916 m_{0}$, perpendicular to the nanowire axis and $m_{z}=m_{t}=0.19 m_{0}$ : two valleys, $\Delta_{x}$, with $m_{l}$ along [100] and two valleys, $\Delta_{y}$, with $m_{l}$ along [010]. The other two valleys, $\Delta_{z}$, have $m_{z}=m_{l}$ along [001] direction and an isotropic confinement mass equal to $m_{t}$ in the nanowire cross section, as shown in Figure 8. The different confinement effective masses give rise to lower subband energies for $\Delta_{x}$ and $\Delta_{y}$ with respect to $\Delta_{z}$ valleys. As a consequence of this and of the larger multiplicity, valleys with low transport mass $m_{z}=0.19 m_{0}$ are overall more populated than those with $m_{z}=0.916 m_{0}$, as shown in the left part of Figure 8 for $V_{\mathrm{G}}=0.5 \mathrm{~V}$ (other values of $V_{\mathrm{G}}$ give similar results). Therefore, $\langle 100\rangle$ devices present 


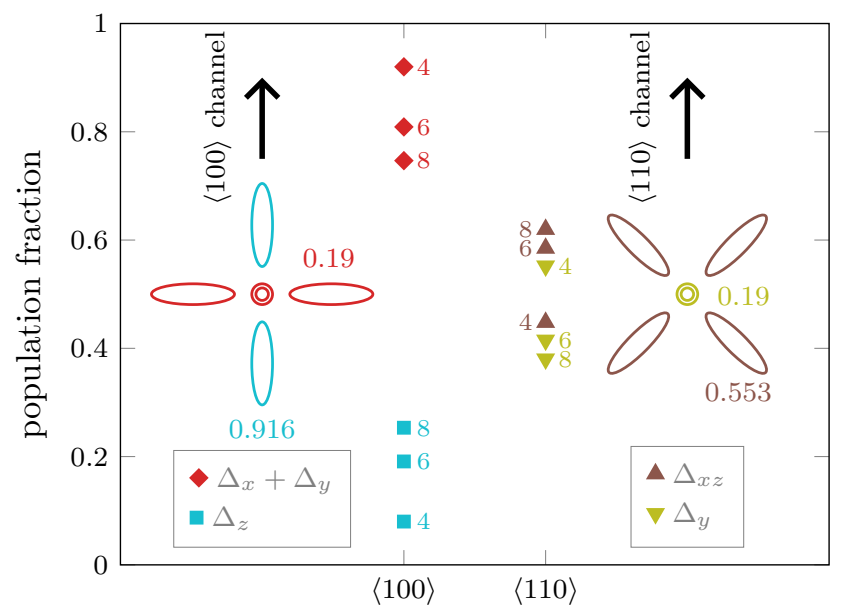

Figure 8: Schematic representation of the six conduction band valleys with respect to channel direction in $\langle 100\rangle$ and $\langle 110\rangle$ nanowires; the values near the ellipses are the transport masses of each set of valleys in units of $m_{0}$. Also shown is the total population of the different sets of valleys in the center of the device for nanowires with different diameters and $V_{\mathrm{G}}=0.5 \mathrm{~V}, V_{\mathrm{D}}=50 \mathrm{mV}$; here the number besides each symbol denotes the nanowire diameter in $\mathrm{nm}$.

a relatively low average value of $m_{z}$.

On the other hand, for $\langle 110\rangle$ nanowires, quantum confinement splits the six degenerate valleys into a set of four-fold degenerate $\Delta_{x z}$ valleys with $m_{l}$ forming an angle of $45^{\circ}$ with the $z$ axis (the transport direction) and two-fold degenerate $\Delta_{y}$ valleys with $m_{l}$ in the confinement plane. In this case, the fundamental subbands correspond to $\Delta_{y}$ valleys because of their larger in-plane mass. However, the larger population in each of those $\Delta_{y}$ valleys do not always generate an overall larger population given the larger multiplicity of $\Delta_{x z}$ valleys. Indeed, in Figure 8 we can see that the overall population of $\Delta_{y}$ valleys is smaller than that of $\Delta_{x z}$ valleys for nanowires with $D=6 \mathrm{~nm}$ and $D=8 \mathrm{~nm}$ and only larger for the narrowest device with $D=4 \mathrm{~nm}$, where confinement is stronger and the subband energy difference prevails. For different bias conditions, the exact values of the valley population differ from those in Figure 8, but the weighted average of $m_{z}$ is always larger than the one obtained for $\langle 100\rangle$ devices, as shown in Figure 7.

We now turn to the spatial charge distribution in a cross section in the middle of 

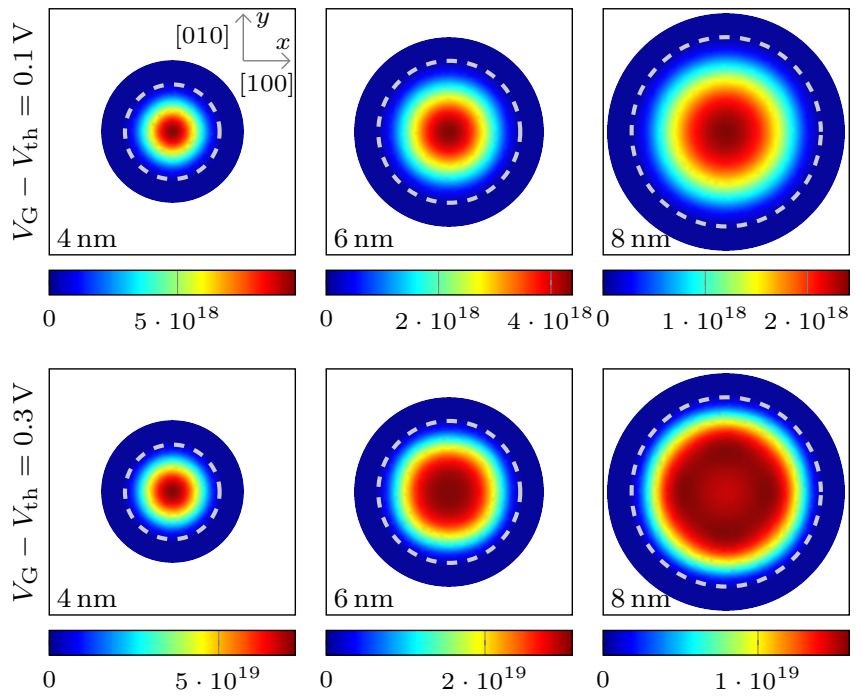

Figure 9: Inversion charge distribution in $\langle 100\rangle$ devices with either $V_{\mathrm{G}}-V_{\text {th }}=0.1 \mathrm{~V}$ or $V_{\mathrm{G}}-V_{\text {th }}=0.3 \mathrm{~V}$ and $V_{\mathrm{D}}=50 \mathrm{mV}$. The dashed line indicates the $\mathrm{Si} / \mathrm{SiO}_{2}$ interface. Units of electron density are $\mathrm{cm}^{-3}$.

the channel. To perform a fair comparison, we plot the electron distribution in different devices with the same values of overdrive voltage $V_{\mathrm{G}}-V_{\mathrm{th}}$. These are shown in Figures 9 and 10 for $\langle 100\rangle$ and $\langle 110\rangle$ orientations, respectively. We can see that in $\langle 100\rangle$ devices the inversion charge is distributed symmetrically. The peak of the distribution corresponds to the geometrical center of the cross section in most of the depicted cases. Only for the larger values of $V_{\mathrm{G}}$ and the widest $D$, the inversion charge moves towards the surrounding gate insulator and four peaks appear along [100] and [010] directions, as can be seen in the bottom right of Figure 9 for $V_{\mathrm{G}}-V_{\text {th }}=0.3 \mathrm{~V}$ and $D=8 \mathrm{~nm}$. On the contrary, Figure 10 shows in-plane asymmetry for $\langle 110\rangle$ devices. In the nanowire with $D=4 \mathrm{~nm}$ (Fig. 10, left), charge distribution extends slightly more in the [101] direction (horizontal in the figure) than in the [010] direction (vertical), while for larger diameters this shape is reversed. As in the case of $\langle 100\rangle$ channel, there is a single peak of charge located at the center of the cross section except for the widest device, $D=8 \mathrm{~nm}$, and for large overdrive voltage, $V_{g}-V_{\text {th }}=0.3 \mathrm{~V}$ (Fig. 10, bottom right), when two main peaks in the [010] direction appear.

The previously observed asymmetry can be quantified by computing the variance 

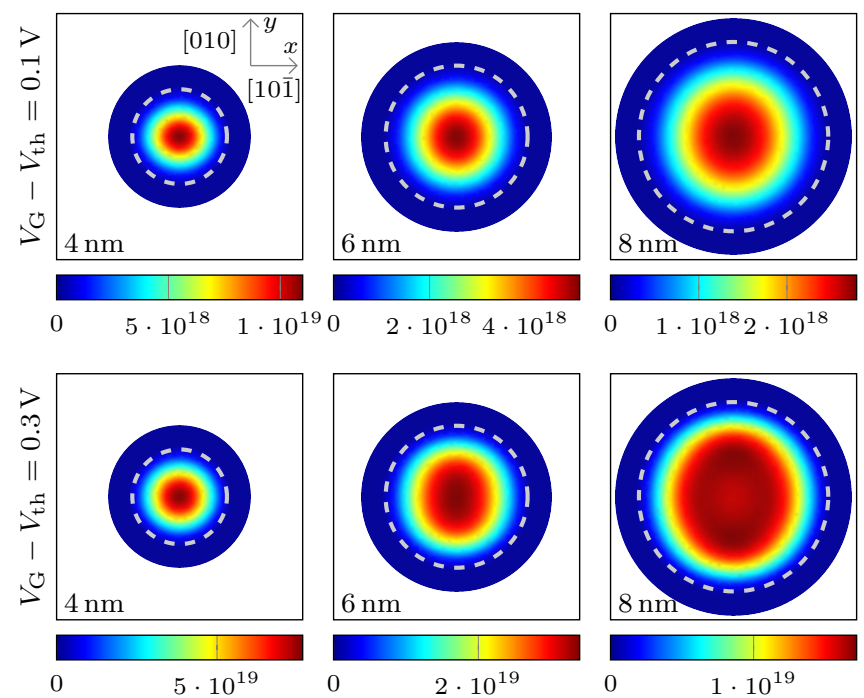

Figure 10: Inversion charge distribution in $\langle 110\rangle$ devices with either $V_{\mathrm{G}}-V_{\text {th }}=0.1 \mathrm{~V}$ or $V_{\mathrm{G}}-V_{\text {th }}=0.3 \mathrm{~V}$ and $V_{\mathrm{D}}=50 \mathrm{mV}$. The dashed line indicates the $\mathrm{Si} / \mathrm{SiO}_{2}$ interface. Units of electron density are $\mathrm{cm}^{-3}$.

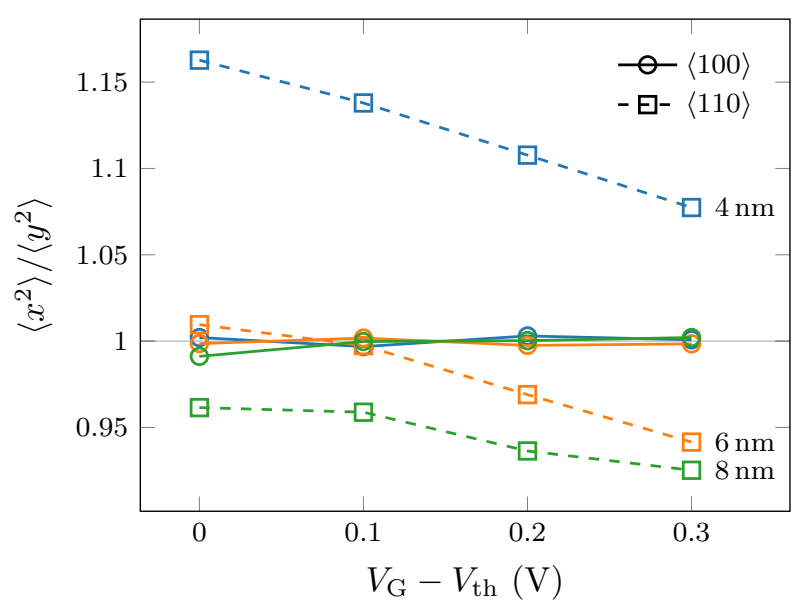

Figure 11: Ratio between the weighted average of $x^{2}$ and the weighted average of $y^{2}$, where the weight is given by the 2D charge distribution. The $x$ direction is ([100] for $\langle 100\rangle$ channel and [101] for $\langle 110\rangle$ channel; the $y$ direction is [010] for both channel orientations. 
of the charge distribution in the horizontal, $x$, direction ([100] for $\langle 100\rangle$ channels and [101] for $\langle 110\rangle$ channels) and the vertical, $y$, direction ([010] for both channel orientations). The ratio of these quantities, $\left\langle x^{2}\right\rangle /\left\langle y^{2}\right\rangle$, is shown in Fig. 11. Such ratio is equal to one, up to the numerical accuracy, for $\langle 100\rangle$ devices. Turning to $\langle 110\rangle$ devices, we can observe that the ratio is larger than unity for $D=4 \mathrm{~nm}$, indicating a greater extension in the horizontal direction, while it is smaller than unity for $D=6 \mathrm{~nm}$ and $D=8 \mathrm{~nm}$, indicating a greater extension in the vertical direction.

The shape of the electron distribution in the different devices can be explained by taking into account the splitting of the six conduction band valleys. For $\langle 100\rangle$ devices, the $\Delta_{x}$ and $\Delta_{y}$ valleys give rise to subbands with the same energy; the corresponding wave-functions are equivalent but rotated by 90 degrees in the cross section plane. The other valleys, $\Delta_{z}$, possess an in-plane isotropic mass: therefore, the corresponding charge distribution is also isotropic. Thus, the cumulative charge density is symmetric for rotations of 90 degrees in the plane, because the contribution of $\Delta_{z}$ is isotropic and those of $\Delta_{x}$ and $\Delta_{y}$ are symmetrically placed. On the other hand, for $\langle 110\rangle$ nanowires both $\Delta_{y}$ and $\Delta_{x z}$ valleys present an asymmetric charge distribution with different shapes: the former elongated along the horizontal direction ([101] $]$ ) and the latter more extended in the vertical direction ([010]). Therefore, the total charge distribution will depend on the relative population of the different valleys, as shown in Fig. 8. For $D=4 \mathrm{~nm}$, the doubly degenerate $\Delta_{y}$ valleys are the most populated ones, and the total charge distribution presents an horizontal shape. For $D=6 \mathrm{~nm}$ and $D=8 \mathrm{~nm}$, instead, the fourfold degenerate $\Delta_{x z}$ valleys represent the larger contribution and the total charge distribution is elongated along the vertical axis.

While the charge distribution depends on channel orientation, the average distance of carriers from the nanowire axis is essentially the same, as depicted in Fig. 12. Here we represent the results of the average radius $r_{\text {avg }}=\sqrt{\left\langle r^{2}\right\rangle}=\sqrt{\left\langle x^{2}\right\rangle+\left\langle y^{2}\right\rangle}$ weighted by the local 2D charge density as shown in Figures 9 and 10. It can be observed that $r_{\text {avg }}$ depends slightly on the applied bias and notably on the nanowire diameter, but not on its orientation. It means that, for a given diameter, the average distance between the charge and the $\mathrm{Si} / \mathrm{SiO}_{2}$ interface does not depend on the nanowire orientation and therefore we can assume that the component of the capacitance due to the spatial distribution 


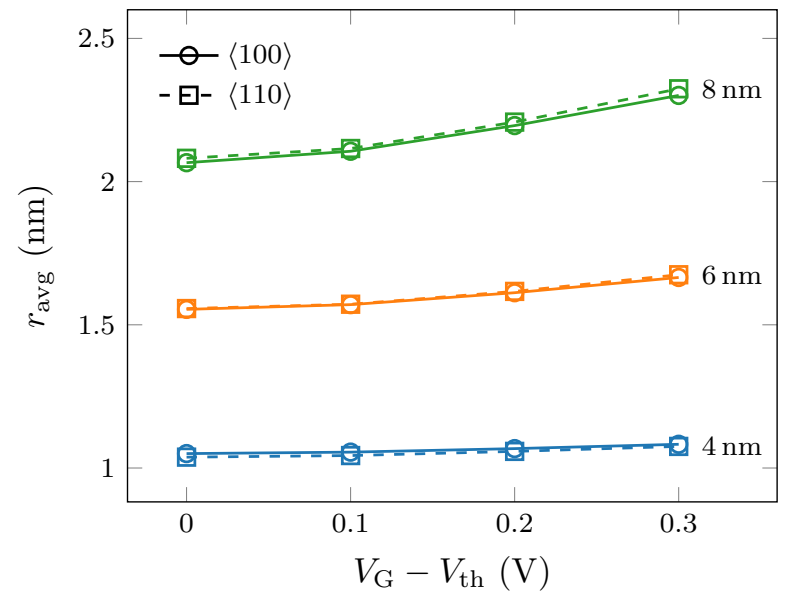

Figure 12: Average distance of the charge from the center of the cross section, measured as the square root of the weighted average of $x^{2}+y^{2}$ where the weight is given by the $2 \mathrm{D}$ charge distribution, as a function of overdrive voltage.

of the inversion charge is the same for $\langle 100\rangle$ and $\langle 110\rangle$ devices. This result explains the behavior depicted in Figure 3, the linear inversion charge in the channel does not depend on the nanowire orientation.

Finally, we look at the scaling behavior of the GAA FETs, and especially its dependence on channel orientation. To do that, we consider nanowires with the same diameter as before but with the channel length, $L_{\mathrm{G}}$ reduced down to $8 \mathrm{~nm}$. For all devices, we computed the transfer characteristics at low and high drain voltages $\left(V_{\mathrm{D}}=50 \mathrm{mV}\right.$ and $V_{\mathrm{D}}=0.5 \mathrm{~V}$, respectively) and we extracted the threshold voltages, shown in Figure 13 as a function of $L_{\mathrm{G}}$. For both $\langle 100\rangle$ and $\langle 110\rangle$ orientations the results are quite similar: as the nanowire diameter is decreased, $V_{\text {th }}$ increases due to the quantum confinement effect, both for low and high drain bias. At the same time, $V_{\text {th }}$ roll-off is observed, i. e. the threshold voltage decreases for shorter values of $L_{\mathrm{G}}$. Narrower devices are more immune to such roll-off: for example, considering $V_{\mathrm{D}}=50 \mathrm{mV}$, for $D=4 \mathrm{~nm}$ a variation of the threshold voltage lower than $10 \mathrm{mV}$ is observed between $L_{\mathrm{G}}=8 \mathrm{~nm}$ and $L_{\mathrm{G}}=14 \mathrm{~nm}$, while for $D=8 \mathrm{~nm}$ a value of $\Delta V_{\mathrm{th}} \sim 65 \mathrm{mV}$ can be estimated in the same $L_{\mathrm{G}}$ range. In any case, the behavior of $\langle 100\rangle$ and $\langle 110\rangle$ devices is very similar, with slightly larger values of $V_{\text {th }}$ for $\langle 110\rangle$ nanowires. 


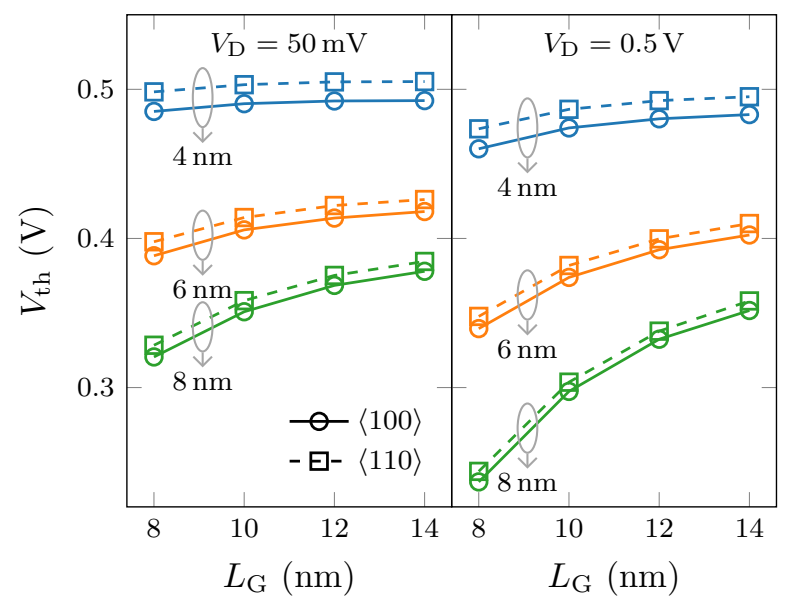

Figure 13: Threshold voltage, computed for different nanowire diameters and orientations, for low and high drain biases, as a function of gate length $L_{\mathrm{G}}$.

Next, for each device we compute the Drain Induced Barrier Lowering (DIBL) as $\left(V_{\mathrm{th}, 1}-V_{\mathrm{th}, 2}\right) /\left(V_{\mathrm{D}, 2}-V_{\mathrm{D}, 1}\right)$, where $V_{\mathrm{th}, 1}$ and $V_{\mathrm{th}, 2}$ are the previously computed values of threshold voltage corresponding to drain voltages $V_{\mathrm{D}, 1}=50 \mathrm{mV}$ and $V_{\mathrm{D}, 2}=0.5 \mathrm{~V}$, respectively. The values of DIBL, shown in Fig. 14, are again slightly larger in the case of $\langle 110\rangle$ devices with respect to $\langle 100\rangle$ ones, but only by a negligible amount. In both cases, if we choose a reference value of $100 \mathrm{mV} / \mathrm{V}$ for the DIBL, at $L_{\mathrm{G}}=10 \mathrm{~nm}$ the devices with $D=4 \mathrm{~nm}$ and $D=6 \mathrm{~nm}$ are within the chosen limit, while at $L_{\mathrm{G}}=8 \mathrm{~nm}$ only the ones with $D=4 \mathrm{~nm}$ fulfill the requirement.

\section{Conclusions}

We compared, through simulations, the performance of GAA n-MOSFETs where the channel is constituted by a cylindrical Si nanowire in either $\langle 100\rangle$ or $\langle 110\rangle$ orientation. The calculations were carried out using our Multi-Subband Ensemble Monte Carlo simulator, which fully captures the quantum properties in the confinement direction including the different valley orientations. Our main conclusion is that $\langle 100\rangle$ devices possess better transport properties: velocity inside the channel, electron mobility and, consequently, drain current are consistently larger than for $\langle 110\rangle$ channels, 


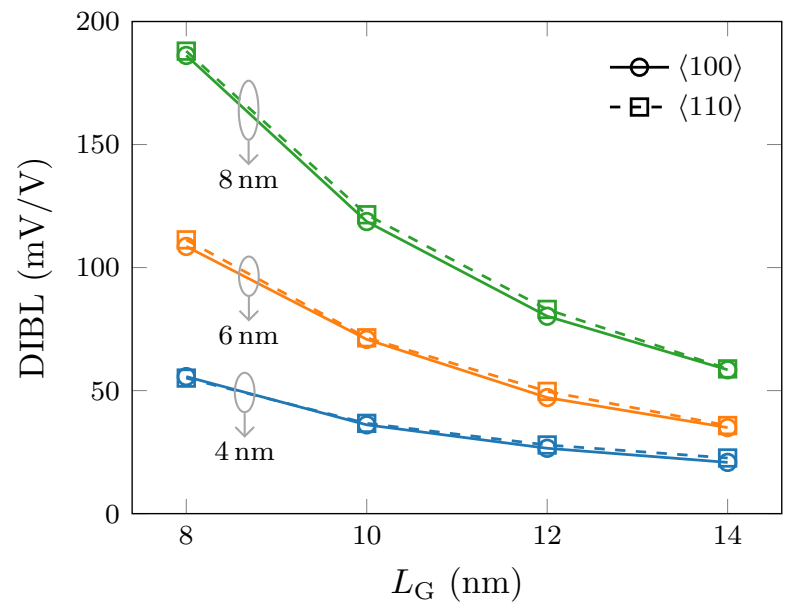

Figure 14: DIBL computed for different nanowire diameters and orientations, as a function of gate length $L_{\mathrm{G}}$.

although not by a large amount. On the contrary, electrostatic behavior is essentially the same for both orientations. This fact is supported by several results: the linear charge density in the middle of the channel, the average radius of electron distribution in the cross section and the scaling behavior only present negligible differences.

We can conclude that narrow GAA FETs with $\langle 100\rangle$ channel can provide larger currents and improved performance. However, if co-integration with bulk devices or FinFET is required so that $\langle 110\rangle$ direction is preferred, the performance loss is moderate and the scaling behavior is essentially the same.

\section{Acknowledgment}

The authors would like to thank the financial support of Spanish Government (TEC201789800-R and PCIN-2015-146) and EU H2020 program (REMINDER, grant agreement No 687931).

\section{References}

[1] N. P. Dasgupta, J. Sun, C. Liu, S. Brittman, S. C. Andrews, J. Lim, H. Gao, R. Yan, P. Yang, 25th Anniversary Article: Semiconductor Nanowires - Synthesis, 
Characterization, and Applications, Advanced Materials 26 (14) (2014) 21372184. doi:10.1002/adma.201305929.

[2] Z. Zeng, F. Triozon, S. Barraud, Y. M. Niquet, A Simple Interpolation Model for the Carrier Mobility in Trigate and Gate-All-Around Silicon NWFETs, IEEE Transactions on Electron Devices 64 (6) (2017) 2485-2491. doi:10.1109/TED.2017.2691406.

[3] J. Fang, S. Chen, W. G. Vandenberghe, M. V. Fischetti, Theoretical Study of Ballistic Transport in Silicon Nanowire and Graphene Nanoribbon Field-Effect Transistors Using Empirical Pseudopotentials, IEEE Transactions on Electron Devices 64 (6) (2017) 2758-2764. doi:10.1109/TED.2017.2695960.

[4] L. Donetti, C. Sampedro, F. G. Ruiz, A. Godoy, F. Gamiz, Multi-Subband Ensemble Monte Carlo simulations of scaled GAA MOSFETs, Solid-State Electronics 143 (2018) 49-55. doi:10.1016/j.sse.2018.02.004.

[5] L. Donetti, C. Sampedro, F. G. Ruiz, A. Godoy, F. Gámiz, Three-dimensional multi-subband simulation of scaled FinFETs, in: 2017 47th European Solid-State Device Research Conference (ESSDERC), 2017, pp. 180-183. doi:10.1109/ESSDERC.2017.8066621.

[6] S. Jin, M. V. Fischetti, T.-w. Tang, Modeling of electron mobility in gated silicon nanowires at room temperature: Surface roughness scattering, dielectric screening, and band nonparabolicity, Journal of Applied Physics 102 (8) (2007) 083715. doi:10.1063/1.2802586.

[7] E. Gnani, S. Reggiani, A. Gnudi, P. Parruccini, R. Colle, M. Rudan, G. Baccarani, Band-Structure Effects in Ultrascaled Silicon Nanowires, IEEE Transactions on Electron Devices 54 (9) (2007) 2243-2254. doi:10.1109/TED.2007.902901.

[8] J. Wang, A. Rahman, A. Ghosh, G. Klimeck, M. Lundstrom, On the validity of the parabolic effective-mass approximation for the I-V calculation of silicon nanowire transistors, IEEE Transactions on Electron Devices 52 (7) (2005) 15891595. doi:10.1109/TED.2005.850945. 
[9] A. Godoy, F. Ruiz, C. Sampedro, F. Gámiz, U. Ravaioli, Calculation of the phonon-limited mobility in silicon Gate All-Around MOSFETs, Solid-State Electronics 51 (9) (2007) 1211-1215. doi:10.1016/j.sse.2007.07.025.

[10] P. Lugli, D. K. Ferry, Degeneracy in the ensemble Monte Carlo method for highfield transport in semiconductors, IEEE Transactions on Electron Devices 32 (11) (1985) 2431-2437. doi:10.1109/T-ED.1985.22291.

[11] A. Pacelli, U. Ravaioli, Analysis of variance-reduction schemes for ensemble Monte Carlo simulation of semiconductor devices, Solid-State Electronics 41 (4) (1997) 599-605. doi:10.1016/S0038-1101(96)00198-0.

[12] D. Jiménez, J. J. Sáenz, B. Iñiguez, J. Suñé, L. F. Marsal, J. Pallarès, Modeling of nanoscale gate-all-around MOSFETs, IEEE Electron Device Letters 25 (5) (2004) 314-316. doi:10.1109/LED.2004.826526.

[13] F. Gamiz, M. V. Fischetti, Monte Carlo simulation of double-gate silicon-oninsulator inversion layers: The role of volume inversion, Journal of Applied Physics 89 (10) (2001) 5478-5487. doi:10.1063/1.1358321. 